\title{
Aplicación de protocolos en enfermería: la mejor barrera contra las infecciones de los catéteres de hemodiálisis
}

\author{
Isabel Crehuet Rodríguez, María Albina Bernárdez Lemus \\ Unidad de Diálisis. Hospital Universitario Río Hortega. Valladolid. España
}

\section{Resumen}

Introducción: La utilización de catéteres venosos tunelizados para hemodiálisis, se ha ido incrementando en los últimos años, aunque su uso no está exento de complicaciones, sobre todo infecciosas, que aumentan la morbi-mortalidad.

Objetivos: Conocer el índice de bacteriemia de nuestra unidad y las complicaciones relacionadas con las mismas.

Material y Método: Se estudió el índice de bacteriemia relacionada con el catéter (número infecciones/número total días-catéterx1000 días catéter) en 30 pacientes portadores de catéteres tunelizados tras una revisión del protocolo del cuidado del catéter. En la segunda mitad del estudio se introdujeron unos nuevos tapones (bioconectores), aunque no era objeto de este estudio la comparación entre los 2 periodos. También se recogieron las complicaciones derivadas de las bacteriemias y el coste económico del tratamiento de dichas bacteriemias.

Resultados: Se obtuvo un índice de bacteriemia de 0,30/1000 días-catéter. No se registraron complicaciones derivadas de la bacteriemia. El coste e económico fue de 127 euros/infección, sin recambio de catéter.

Conclusión: A la vista de estos resultado podemos concluir que la tasa de BRC de nuestra unidad es baja, y que en ello, confluyen varios factores: la utilización estricta de un protocolo de manipulación de los catéteres tunelizados, el conocimiento y seguimiento generalizado del mismo

\begin{tabular}{|c|}
\hline Correspondencia: \\
Isabel Crehuet Rodríguez \\
Unidad de Diálisis. Hospital Universitario Río Hortega \\
C/ Dulzaina $n^{\circ} 2.47012$ Valladolid \\
E-mail: crebel@hotmail.com \\
\hline
\end{tabular}

por todo el personal de enfermería, en el que destacaríamos realizar cualquier manipulación con 2 profesionales; y sobre todo, la concienciación de todo el grupo, de que la primera prevención de las infecciones relacionadas con los catéteres de HD, son las medidas de barrera.

PALABRAS CLAVE: bacteriemia relacionada con el catéter; infección; hemodiálisis; cuidados de enfermería.

Implementation of protocols by nursing: The best barrier against infections of hemodialysis catheters

\section{Abstract}

Introduction: Using tunneled venous catheters for hemodialysis has increased in recent years, although its use is not free of complications, especially infectious, and increases morbi-mortality.

Objectives: To know the rate of bacteremia of our unit and the related complications.

Material and Method: The rate of catheter relatedbacteremia was determined (number of infections / total number of catheter-days x 1000 catheter-days) in 30 patients with tunneled catheters after a revision of the catheter care protocol. New plugs (bioconnectors) were introduced in the second half of the study, although the comparison between the two periods was not an objective. The complications resulting from the bacteremia and the economic cost of the treatment of it were also recorded.

Results: The rate of bacteremia was $0.30 / 1.000$ catheter-days. There were no complications resulting from bacteremia. The economic cost was 127 euros/infection, without catheter replacement. 
Conclusion: In view of these results we can conclude that the rate of dialysis catheter-related bacteriemia in our unit is low. Several factors converge on this: the strict use of a protocol for the management of tunneled catheters and the knowledge and generalized monitoring of it, by all nursing staff. About this protocol, we would emphasize carrying out any manipulation on the catheter with 2 professionals and, above all, the awareness of the whole group, that the the barrier measures are the first prevention of infections related to HD catheters.

KEYWORDS: catheter-related bacteremia; infection; hemodialysis; nursing care.

\section{Introducción}

Muchos han sido los avances producidos, en pocos años, en las terapias sustitutivas renales: monitores más complejos y seguros, capaces de realizar nuevas técnicas además de la hemodiálisis (HD) convencional como la hemodiálisis de alto flujo, hemodiafiltración libre de acetato (AFB), hemodiafiltración en línea (HDF ON-LINE), etc.; sin embargo, el acceso vascular (AV), a pesar de ser una condición sine qua non para realizar estas técnicas", sigue siendo el "talón de Aquiles" en los pacientes sometidos a estas terapias. Según las guías clínicas de accesos vasculares, los catéteres venosos centrales tunelizados (CVCT) deberían ser la última opción de acceso vascular para realizar HD por detrás de cualquier tipo de fístula arteriovenosa (FAV) 2,3 . Está comprobado que ser portador de un catéter para HD se asocia a un aumento de la morbi-mortalidad ${ }^{4}$ y que las complicaciones que las complicaciones de este $\mathrm{AV}$ son la primera causa de ingreso hospitalario, lo que supone un elevado coste para el sistema sanitario. A pesar de ello, estamos asistiendo en nuestras unidades a un aumento considerable de portadores de catéter, debido al cambio en el perfil del paciente en HD: edad avanzada, previsión de una supervivencia no muy larga por comorbilidad añadida, tiempo de espera corto para un trasplante de vivo, descanso de la técnica de diálisis peritoneal, etc. Una de las principales complicaciones relacionadas con la utilización de estos CVCT son las infecciones, que constituyen la segunda causa de muerte por detrás de las enfermedades cardiovasculares ${ }^{5}$.

La bacteriemia relacionada con el catéter (BRC) puede ser un proceso bastante grave dependiendo del agente que lo produzca; por ejemplo, en el caso del Staphylococcus aureus ha llegado en ocasiones a producir infecciones me- tastásicas muy graves: endocarditis (con una mortalidad cercana al 50\%), osteomielitis, espondilodiscitis, etc ${ }^{6,7}$.

Ante este reto, en las Unidades de Diálisis se vienen extremando las medidas de asepsia por parte de enfermería, en el manejo y cuidados de los $\mathrm{CVCT}^{8}$, intentando aproximarse al programa Bacteriemia Zero, promovido desde la Universidad de John Hopkins, en EEUU, por el doctor Peter Provonost quien tras la aplicación de este programa en 103 Unidades de Cuidados Intensivos (UCI) consiguió reducir las bacteriemias relacionadas con catéteres venosos centrales a prácticamente cero. En España, la Agencia de Calidad del Ministerio de Sanidad, Servicios Sociales e Igualdad, junto con la OMS ha promovido este proyecto que ha sido liderado por la Sociedad Española de Medicina Intensiva, Crítica y Unidades Coronarias (SEMICYUC) ${ }^{9}$, implantándolo en varias UCIs de nuestro país con resultados altamente satisfactorios. Resulta complicado aplicar este programa en Unidades de HD por las lógicas diferencias de situación clínica, contexto y pacientes, ya que éstos, a diferencia de los pacientes de UCI, reciben el tratamiento de forma ambulatoria?.

Conociendo estas premisas, y basando nuestros protocolos en las principales medidas del programa Bacteriemia Zero, nos planteamos realizar el presente estudio sobre las BRC en nuestra unidad, con los siguientes objetivos:

- Conocer el índice de BRC y las complicaciones derivadas de las misma.

- Averiguar el coste económico del tratamiento de dichas BRC, en cuanto a material fungible, en el momento del estudio.

\section{Material y Método}

Se realizó un estudio transversal retrospectivo con una muestra de 30 pacientes en programa de HD crónica cuyo acceso vascular era un CVCT. Tras una revisión de los protocolos de actuación sobre los catéteres en nuestra unidad (Anexo 1) y una vez aplicados los mismos durante un periodo total de 1000 días, estudiamos las BRC producidas en estos pacientes durante dicho periodo, las complicaciones que se produjeron, si desembocaron en infecciones metastásicas (endocarditis, osteomielitis, etc.) si requirieron recambio del catéter e incluso si provocaron la muerte, fuera cual fuera la vía de entrada de los gérmenes. En la segunda mitad de este periodo de estudio se modificaron los tapones para cerrar los catéteres, siendo los nuevos unos ta- 
pones especialmente diseñados para los catéteres de HD (bioconectores, $\operatorname{Tego}^{\circledR}$ ). La diferencia entre ambos tapones radica en que los bioconectores tienen válvula antirreflujo y sólo se recambian una vez por semana, y los tapones habituales que utilizábamos eran de un solo uso, con rosca luer-lock y se recambiaban en cada sesión de HD.

La manipulación del catéter implicaba exponer al exterior la luz de las ramas del mismo como mínimo dos veces por sesión: conexión y desconexión del monitor de HD. Si en algún momento el catéter llevaba asociado algún proceso de disfunción, las manipulaciones sobre el mismo aumentaban considerablemente. Además, el orificio de salida del catéter (OSC) también es expuesto al exterior en cada sesión de HD, ya que se cura en cada una de ellas.

Consideramos BRC la aparición de fiebre en un paciente portador de un CVCT para HD, con hemocultivos positivos y sin otro foco de infección aparente. El tratamiento de la misma consistió, en el momento en que el paciente refirió síntomas (fiebre durante el periodo inter o intradiálisis), realizar hemocultivos extraídos de ambas ramas del catéter, antibioterapia de amplio espectro de forma empírica (Vancomicina) hasta obtener el resultado de los mismos, y a partir de entonces antibioterapia sobre antibiograma que se mantuvo durante un periodo que osciló entre 4-6 semanas y que se administró de manera ambulatoria al finalizar la sesión de HD correspondiente. En ocasiones, además, si los síntomas persistían durante más de 48-72 horas se requirió la retirada del catéter e inserción de otro nuevo, a ser posible, en un lugar distinto al anterior.

Todos los CVCT fueron implantados en Radiología Vascular, bajo control radioscópico, siguiendo las medidas universales de asepsia, sin profilaxis antibiótica y sellándolos con heparina al 5\%.

El índice de BRC se calculó con la siguiente fórmula:

Índice de $\mathrm{BRC}=\mathrm{N}^{0}$ infecciones $/ \mathrm{N}^{\circ}$ total días-catéter $\times 1000$ días-catéter

Además, se calculó el coste económico del tratamiento de una BRC: material fungible, fármacos, recambio de catéter e incluso el día de ingreso para recambiarlo.

Todos los pacientes fueron informados de la realización del estudio y dieron su consentimiento verbal. La información recogida fue tratada con arreglo a la actual Ley
Orgánica 15/1999 de 13 diciembre, sobre "Protección de datos de carácter personal", y con el único fin del desarrollo de esta investigación.

El análisis estadístico de los datos se realizó con el programa SPSS 15.0 para Windows. Las variables se expresaron como la media junto con la desviación estándar para las edades y como la mediana más el rango intercuartílico para el tiempo de permanencia en HD.

\section{Resultados}

De los 30 pacientes incluídos en el estudio, 10 eran mujeres. La media de edad fue de $69,2 \pm 13,97$; siendo la mediana de permanencia en HD de $38,5(22,25-70,00)$ meses.

De los 30 catéteres estudiados, 29 eran de carbotano (Palindrome ${ }^{\mathrm{TM}}$ ), excepto uno que era de poliuretano, tipo Split; 22 estaban situados en vena yugular interna derecha, de los cuales uno estaba situado en vena yugular interna izquierda, pero lo consideramos como derecha pues el paciente presentaba un "situs inversus", 3 en vena femoral derecha y otro en vena femoral izquierda.

En el periodo de estudio se registraron $6 \mathrm{BRC}$, de acuerdo a los criterios de infección establecidos previamente; fueron 19.667 el número total de días-catéter de todos lospacientes incluídos, con lo que el índice de BRC fue el siguiente:

Índice de $B R C=6 / 19667 \times 1000$ días-catéter $=0,30 / 1000$ días catéter

Para averiguar la posible influencia en BRC, de la utilización de los bioconectores, se analizaron las BRC antes y después del uso de los mismos. Durante los primeros 500 días en los que se utilizaron tapones convencionales de un solo uso, contabilizamos un número total de 8.407 días-catéter siendo 4 las infecciones producidas con lo que el índice de bacteriemia se situó en 0,60/1000 días-catéter. Durante el periodo en que utilizamos los nuevos tapones o bioconectores, alcanzamos un total de 11.260 días-catéter y se produjeron dos infecciones con lo que el índice de BRC fue de 0,28/1000 días-catéter.

Se registraron 10 infecciones del OSC durante el periodo estudiado. De ellas 4 se produjeron en el mismo paciente: 3 por Staphylococcus aureus y 1 por Esche- 
richia coli, siendo esta última la única que originó una de las BRC por el mismo germen, y que requirió tratamiento por vía sistémica, además de por vía tópica. Las otras 6 se produjeron en otros 6 pacientes y con gérmenes diferentes, predominando también Staphylococcus aureus (4), y Staphylococcus coagulasa negativa (2), aunque ninguna originó bacteriemia. Todas estas infecciones del OSC fueron tratadas tópicamente, respondiendo bien al tratamiento. En ningún caso hubo que recambiar el catéter ni se produjeron complicaciones.

Respecto al aspecto económico de las infecciones, en el momento del estudio, en nuestro centro supone sobre 127 €/infección, calculando únicamente el material fungible. Resultó muy difícil cuantificar el coste de los tiempos de trabajo de microbiólogos, farmacéuticos, nefrólogos, enfermeros, etc. El coste aumentaría considerablemente si el paciente tuviera que ser sometido a un recambio de catéter (el precio medio de los catéteres utilizados en nuestro centro es de $500 €$ ) ya que además implicaría un ingreso hospitalario cuyo coste supone $408,74 €$ /día en este momento (Tabla 1).

Tabla 1. Coste de una BRC en nuestro centro, en el momento del estudio.

\section{COSTE ECONÓMICO DE UNA BRC}

\begin{tabular}{l|r} 
Equipo infusión bomba & $3,41 €$ \\
Aguja de carga & $0,04 €$ \\
Jeringa de $10 \mathrm{cc}$ & $0,04 €$ \\
Guantes estériles & $0,26 €$ \\
Guantes no estériles & $0,03 €$ \\
Suero fisiológico 250 cc & $0,76 €$ \\
Vancomicina lgr. & $1,61 €$ \\
TOTAL (por sesión) & $6,15 €$ \\
TOTAL (18 sesiones) & $110,70 €$ \\
Perfalgán lgr. (1 dosis) & $0,64 €$ \\
Frascos hemocultivo (4) & $16,11 €$ \\
TOTAL (por tratamiento. de 18 sesiones) & $127,45 €$
\end{tabular}

(+ pruebas de laboratorio)

${ }^{*}$ Si hubiera que recambiar el catéter:

Catéter

$500 €$

Ingreso hospitalario/ día

$408,74 €$

\section{Discusión}

Los profesionales de las unidades de diálisis son muy conscientes de la gravedad que suponen las infecciones de los catéteres para los pacientes en HD, lo que implica esforzarse y poner un celo especial en los cuidados de los mismos: revisión de protocolos, comprobación de la correcta aplicación de los mismos, educación sanitaria, etc. En nuestra unidad hemos obtenido una tasa de BRC muy baja en el periodo estudiado, que de acuerdo a los rangos que plantea Beathard $G$ y Urbanes $A$ en su estudio ${ }^{10}$, sería una tasa excelente. Varios son los factores que contribuyen en la obtención de estos resultados: protocolos, en los que se incluye la conexión del CVCT al circuito sanguíneo entre dos profesionales, para así aplicar las máximas medidas de asepsia, protocolos sometidos a revisiones en función de los materiales nuevos que se van incorporando y de la evidencia científica, personal debidamente entrenado para realizar la técnica, tiempo necesario para manejar los catéteres, etc. resultados que concuerdan con el estudio de Albalate M. y cols. en el que obtuvieron unas tasas de BRC muy bajas incluso sin el uso de bioconectores $^{11}$. Es evidente, que en nuestros resultados las tasas de BRC disminuyen de forma importante en el segundo periodo, en el que se introduce el uso de los bioconectores, algo que pudimos observar en otro estudio, en el que aun partiendo de tasas de BRC también muy bajas, se encontraron diferencias significativas entre utilizar o no estos dispositivos ${ }^{12}$; sin embargo, los resultados a este respecto son algo contradictorios. Algunos autores, como Martínez MA y cols. no encontraron diferencias significativas en la reducción de bacteriemias al usar los bioconectores, quizás porque presentaban unas tasas de BRC muy bajas y el tiempo estudiado fue escaso $0^{13}$. Mcafee y cols. en su estudio en una unidad pediátrica reportaron resultados de disminución de un $50 \%$ en las tasas de infección de los catéteres ${ }^{14}$ al igual que el de Cobo JL. y cols. en el que sí encontraron diferencias muy significativas al usar estos bioconectores, ya que partían de tasas altas de BRC ${ }^{15}$. En nuestra experiencia, aun sin utilizar los bioconectores en el primer periodo de estudio, la tasa de BRC fue menor de 1/1.000 días-catéter, estando también en el rango de la excelencia. El hecho de disminuir el número de exposiciones al exterior de las luces del catéter, a priori, debe hacer pensar que reduce el número de infecciones. Sin embargo, no ha sido objeto de este estudio la posible diferencia de bacteriemias entre el uso de ambos tapones. Hemos considerado estos dispositivos como una barrera más de prevención de la infección como pueden ser el uso de material estéril para manipular el catéter, realizar la conexión entre dos personas, utilizar la clorhexidina como agente desinfectante etc. porque la vía de entrada de gér- 
menes se produce tanto por las ramas del catéter como por el orificio de salida del mismo a través de la piel.

Otro factor importante a tener en cuenta es la ratio enfermera/paciente utilizada en nuestra unidad. En nuestra unidad la ratio enfermera/paciente fue de $1 / 4$ como máximo, siguiendo las recomendaciones de las Guías de la Sociedad Española de Nefrología, independientemente del acceso vascular que tenga el paciente. No en todas las unidades de HD las ratios son las mismas; en algunas es de $1 / 5$, incluso llegan hasta $1 / 6$, mientras que en otras es de 1 enfermera/3-4 pacientes y cuidando que los pacientes que atiende la misma enfermera porten FAVn o FAVp y CVCT en una relación de 2 ó 3 FAV/I CVCT, tal como describieron en su estudio Cárcamo J. y cols. para aplicar unos cuidados de calidad a los pacientes ${ }^{16}$.

Respecto a los costes directos e indirectos de las infecciones, no hemos encontrado referencias con quien comparar el coste de las mismas, y sobre todo, tal como hemos reflejado en los resultados, existen grandes diferencias en el coste de la infección de CVCT dependiendo de que evolucione bien con el tratamiento antibiótico o necesite un recambio y su correspondiente día de ingreso hospitalario (Tabla 1).

Por último, no podemos olvidar, al margen de la alteración de la salud que supone una infección en un paciente de $H D$, el malestar emocional y/o psicológico que va a sufrir este paciente. En nuestra unidad observamos que, una vez superado el primer día de la infección, sin duda el peor por el malestar físico que sienten, tienen además un malestar emocional derivado de los tiempos añadidos a su sesión de diálisis, para la administración del tratamiento antibiótico durante unas 12-18 sesiones. Sin embargo, el tiempo que tarda enfermería en manipular un catéter adecuadamente oscila entre 10-15 minutos para conectar al monitor y otros tantos para desconectar. La enfermería de las unidades de diálisis, no puede obviar que los accesos vasculares son limitados y que, aunque existan nuevos antibióticos, nuevos materiales o productos sanitarios (antisépticos, dispositivos, soluciones para el sellado, etc) que frenan las infecciones, la principal barrera contra la bacteriemia consiste en la buena práctica enfermera para diseñar y aplicar unos protocolos adecuados. En nuestro caso, el hecho de tener una tasa tan baja de BRC nos permite situarnos, en el nivel de la excelencia en cuanto a los cuidados de los CVCT. No obstante, alcanzar estos niveles no debe hacernos bajar la guardia.

A la vista de estos resultados podemos concluir que la tasa de BRC de nuestra unidad es baja, y que en ello, confluyen varios factores: la utilización estricta de un protocolo de manipulación de los catéteres tunelizados, el conocimiento y seguimiento generalizado del mismo por parte de todo el personal de enfermería, en el que destacaríamos realizar cualquier manipulación con 2 profesionales; y sobre todo, la concienciación de todo el grupo, de que la primera prevención de las infecciones relacionadas con los catéteres de HD, son las medidas de barrera. Además, a las medida de barrera clásicas, añadir el uso de bioconectores que se recambian una vez por semana, parece influir claramente en la disminución de las BRC.

\section{Agradecimientos}

A todos los pacientes por su colaboración.

A todo el personal que hace posible, con su trabajo, prestar estos cuidados.

Recibido: 29 diciembre 2017

Revisado: 5 febrero 2018

Modificado: 24 mayo 2018

Aceptado: 30 junio 2018

\section{Bibliografía}

1. Roca Tey R. El acceso vascular para hemodiálisis: la asignatura pendiente. Nefrología. 2010;30(3):280-7.

2. Rodríguez JA, González $E$, Gutiérrez JM, Segarra A, Almirante B, Martínez MT et al. Guías de acceso vascular en hemodiálisis (Guías S.E.N.). Nefrología. 2005;25(Supl 1):S3-97.

3. National Kidney Foundation. KDOQI Clinical Practice Guidelines and Clinical Practice Recommendations for 2006 Updates: Hemodialysis Adequacy, Peritoneal Dialysis Adequacy and Vascular Access. Am J Kidney Dis. 2006;48(Supl 1):S1-322.

4. Fernández-Gallego J, López V, Martín M.A, Toledo $R$. El catéter venoso central crónico tunelizado aumenta la mortalidad en hemodiálisis. Nefrología. $2005 ; 25(6): 720$. 
5. Registro español de Enfermos Renales. Informe 2012 de diálisis y trasplante renal en España. Disponible en: www.senefro.org.

6. Liangos 0 , Gul A, Madias NE, Jaber BL. Long-Term Management of the Tunneled Venous Catheter. Seminars in Dialysis. 2006;19:158-64.

7. Miler LM, Clark E, Dipchand C, Hiremath S, Kappel $\mathrm{J}$, Kiaii $\mathrm{M}$ et al. Hemodialysis Tunneled Catheter Related Infections. Canadian Journal of Kidney Health and Disease. 2016;3:1-11.

8. Rosenblum A, Wang W, Ball LK, Latham C, Maddux FW, Lacson EJr. Hemodialysis Catheter Care Strategies: A Cluster Randomized Quality Improvement Initiative. Am J Kidney Dis. 2014;63(2):259-67.

9. Página web del Ministerio de Sanidad, Servicios Sociales e Igualdad. [Consultado Abril 2014]. Disponible en: http://www.msssi.gob.es/organizacion/sns/ planCalidadSNS/bacteriemia

10. Beathard G. A., Urbanes A. Infection associated with tunneled hemodialysis catheter. Semin Dial. 2008;21:528-38.

11. Albalate $M$, Pérez García $R$, De Sequera $P$, Alcázar $R$, Puerta M, Ortega M, Mossé A, Crespo E. ¿Hemos olvidado lo más importante para prevenir las bacteriemias en pacientes portadores de catéteres para hemodiálisis? Nefrología. 2010;30(5): 573-7.

12. Crehuet Rodríguez I, Bernárdez Lemus M A, Ramírez Crehuet M, Méndez Briso-Montiano P, Ruiz-Zorrilla López C. Bioconectores: ¿Son realmente eficaces en la prevención de las bacteriemias relacionadas con el catéter permanente para hemodiálisis?. Rev Soc Esp Enferm Nefrol. 2013;16(4): 235-40.

13. Martínez Aranda MA, Arribas Cobo $P$, Ruiz Álvarez $A$, Rodríguez Estaire J, Callejo Cano I, Díaz de Argote P et al. Eficacia del uso de bioconectores para los catéteres centrales de hemodiálisis. Rev Soc Esp Enferm Nefrol. 2011;14(2):106-11.
14. Mcafee N, Seidel K, Watkins S, Flynn JT. A continuous quality improvement project to decrease hemodialysis catheter infections in pediatric patients: use of a closed luer-lock access cap. Nephrol Nurs J. 2010; 37(5):541-44.

15. Cobo Sánchez JL, Sainz Alonso RA, Vicente Jiménez MY, Cepa García H, Pelayo Alonso R, Menezo Viadero $R$ et al. Estudio comparativo de incidencia de bacteriemias relacionadas con el catéter de hemodiálisis: bioconectores frente a conexión directa. Rev Soc Esp Enferm Nefrol. 2011;14(4):230-5.

16. Cárcamo Baena J, Salgueira Lazo M, Gómez Castilla C, Rodríguez Pérez MA, Tienda Moreno M, Rico Castillo $\mathrm{C}$ et al. Modelo de manejo multidisciplinar de catéteres permanentes tunelizados: resultados a 5 años Rev Soc Esp Enferm Nefrol. 2012;15(2): 138-44.

\section{ANEXO 1}

Aspectos fundamentales en el manejo de los catéteres venosos centrales en nuestra unidad.

Al margen de los protocolos propios de cada técnica: conexión-desconexión, disfunción, etc. consideramos que lo más relevante en cuanto a la prevención de la BRC es:

- Cumplir escrupulosamente el protocolo de higiene de manos: lavado adecuado, uñas cortas y sin pintar, retirar anillos, pulseras, relojes, etc..

- Realizar el procedimiento entre dos personas: una para manejar la parte estéril otra para la no estéril.

- Aplicar las medidas universales de asepsia: mascarillas, uso de material estéril: paños, guantes, etc. en cualquier manipulación sobre el catéter: inversión de ramas, lavados, toma de muestras, etc. desinfectando los terminales con clorhexidina al $2 \%$, así como sobre el orificio de salida del catéter.

- Curar por separado el OSC. Si hay secreciones o exudados tomar muestra para cultivo.

- Utilizar campana estéril para extracción de muestras sanguíneas a través de los terminales del catéter (con jeringa si se trata de hemocultivos).

Este artículo se distribuye bajo una Licencia Creative Commons Atribución-NoComercial 4.0 Internacional. https://creativecommons.org/licenses/by-nc/4.0/ 